\title{
THEORETICAL INVESTIGATION OF STRESSES DISTRIBUTIONS IN HOLLOW SANDCRETE BLOCKS DUE TO VARIATION OF CAVITY SIZES AND NUMBER WEB THICKNESS
}

\author{
J. C. Agunwamba ${ }^{1}$, J. C. Ezeokonkwo ${ }^{2,}{ }^{*}$, M. E. Onyia ${ }^{3}$, E. Ojiogu ${ }^{4}$ and A. Amadou ${ }^{5}$ \\ 1,2,3Department OF Civil ENGinEeRING, UNIVERSity OF NigERIA, NSUKKA, ENUGU STATE. NIGERIA

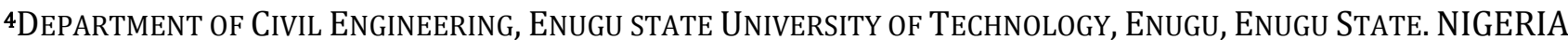 \\ ${ }^{5}$ FACULTÉ DES SCIENCES, UNIVERSITÉ DE DosSo, DOSSO, NIGER \\ E-mail-addresses: 1jonah.agunwamba@unn.edu.ng, ${ }^{2}$ josephat.ezeokonkwo@unn.edu.ng, \\ 3ebieonyia64@yahoo.com,4ojiogue@yahoo.com,5adamou.amadou@unn.edu.ng
}

\begin{abstract}
The relationship between cavity size and number on one hand and combinations of web thicknesses were investigated theoretically. The least number of cavities was 4 for end-web to centre web ratio of 1:2 at constant shell thickness with the least web thickness equal to $12.5 \mathrm{~mm}$. The effects of the number of cavities and web thickness on strength were also investigated. The test thin plate distributes the load on the block and the hollow block is regarded as a two way slab. One of the principal findings is that the variation of maximum stresses with the number of cavity is Gaussian for end-web to centre-web ratio of 1:1 but polynomial for 1:2. In addition, the lowest stress is induced when the end to centre ratio is one. The maximum stress, $\left(21.5 \mathrm{~N} / \mathrm{mm}^{2}\right)$ occurred at the cavity length (a*) of $125 \mathrm{~mm}$ for 3 cavities while the end (end centre) web thickness ( $t$ ) was 25mm respectively. In order words, this combination should be avoided as it will lead to creation of highest stress in the hollow sandcrete blocks. The configuration which will result in least stress $\left(0.5 \mathrm{~N} / \mathrm{mm}^{2}\right)$ is $(\mathrm{a}, \mathrm{N}, t)=(50 \mathrm{~mm}, 4 \mathrm{~mm}, 50 \mathrm{~mm})$. However, this combination also gave a high maximum stress $\left(6.9 \mathrm{~N} / \mathrm{mm}^{2}\right)$ in a web, implying that the right choice of configuration should be rather the one that creates the smallest set of maximum stresses in all members. Overall, the best configuration is 2 cavities with web thickness $50 \mathrm{~mm}$ for an end web to centre web ratio of 1:1 (3.3N/mm²). The corresponding values for 1: 2 ratios are 3 cavities with $50 \mathrm{~mm}$ shell thickness (4.8N/(mm $\left.{ }^{2}\right)$. The method presented showed meaningful insight could be investigating theoretically.
\end{abstract}

Keywords: hollow sandcrete blocks, cavity size, cavity number, series arrangements, parallel arrangements, stresses

\section{INTRODUCTION}

Sandcrete blocks, moulded into different sizes [1], are often used as wall construction materials for building. Building industries in Nigeria often produce two-cell hollow sandcrete blocks $[2,3]$, but it is necessary to investigate other configurations and sizes, especially as they affect the compressive strength of sandcrete.

Some research works had been done on the relationship between cavity characteristics, web thickness and strength. The variations in cavity volumes, shell thickness, web thickness and cavity shape affect the compressive strength of hollow blocks. Udoeye et al. examined the influence of geometry on the compressive and tensile strength of laterized concrete and noted that it has significant effect [4].

It has been shown by Ezeokonkwo that centre-web to end-web ratio of unity does not give hollow sandcrete blocks of maximum compressive strength $[5,6]$.

Ezeokonkwo also investigated two-cell hollow sandcrete blocks and stated that the size of the block cavity would affect its compressive strength [5]. His results showed that there exists an optimum combination of cavity volume with centre-web to end -web ratio that would give the maximum strength.

It is obvious from literature that not much investigation had been done on the relationship between the size and number of cavities and web 
thickness on one hand and their effect on the compressive strength of hollow sandcrete hollow blocks on the other. Hence, this research paper is aimed at determining the theoretical optimum cavity size, web thickness and cavity number that would yield the highest strength. The results would help reduce the cost, labour and time necessary to investigate all the configurations and sizes of cavity that would give the best strength.

\section{STRESS ANALYSIS}

In order to determine the stress distribution on the hollow blocks of varying number of cavities, the webs are regarded as the supports. The test thin plate distributes the load on the block and the hollow block is regarded as a two way slab. The details of the analysis are shown in the Appendix. The coefficients were determined from a design manual [7]. The variation of maximum and minimum stresses with cavity length and number of cavity are shown in Figures 1 to 8 for cases where end-web to centre-web ratios is $1: 1$ and $1: 2$.

\section{RESULTS AND DISCUSSIONS}

\subsection{Effects of Web to Centre-web Ratios}

The effects of end-web to centre-web ratios on the maximum and minimum stresses developed in the block are shown in Figures 1 to 4 and 5 to 8 respectively. The overall maximum stress developed is $22 \mathrm{~N} / \mathrm{mm}^{2}$ (Fig. 2) at an end-web to centre-web ratio of one as opposed to the value of $14.3 \mathrm{~N} / \mathrm{mm}^{2}$ for a ratio of $1: 2$ for $22.5 \mathrm{~mm}$ web thickness (Fig. 1). Generally, higher stresses are induced at a ratio of 1:1 than 1:2 for the same number of cavities and web thickness which is in line with the findings of Ezeokonkwo [5].

In addition, as expected the maximum stresses occur when the number of cavities are 2 and 3 for the ratios of 1: 2 and 1:1 respectively. Variation of maximum stress with number of cavity is polynomial for the ratio of $1: 2$ while for $1: 1$ it is approximately Gaussian.

The lowest of the maximum stresses is $4.8 \mathrm{~N} / \mathrm{mm}^{2}$ for the $1: 2$ ratio and all web thicknesses whereas for the ratio of $1: 1$ the values were approximately $7.53 \mathrm{~N} / \mathrm{mm}^{2}, 6.53 \mathrm{~N} / \mathrm{mm}^{2}, \quad 5.53 \mathrm{~N} / \mathrm{mm}^{2}$ and $3.3 \mathrm{~N} / \mathrm{mm}^{2}$ corresponding to the web thicknesses equal to $22.5 \mathrm{~mm}, 25 \mathrm{~mm}, 37.5 \mathrm{~mm}$ and $50 \mathrm{~mm}$ respectively (Figure 2). This shows that the higher the thickness of the web the lower the induced stress.

Another obvious result is that the same stress desired could be achieved at various combinations of the number of cavities and web thickness. For instance, in the case of 1:2 ratios (Figure 1), the same lowest maximum stress could be obtained by creating 3, 5, 7 and 8 cavities with web thickness of $50 \mathrm{~mm}, 37.5 \mathrm{~mm}$, $25 \mathrm{~mm}$ and $22.5 \mathrm{~mm}$ respectively.

The effect of the end-web to centre-web ratio on the lowest induced stress is evident in Figures 3 and 4. The stresses reduced as the web thicknesses and cavities increase. For the 1:1 ratio, the global minimum is $0.5 \mathrm{~N} / \mathrm{mm}^{2}$ for 4 cavities and $50 \mathrm{~mm}$ thickness. In order words, the optimum minimum stress is induced when 4 cavities are created for endweb to centre-web ratio of 1:1 with thickness of $50 \mathrm{~mm}$ for the ranges of values considered. However, high stresses occur in some members with 4 cavities implying that the configuration with the least stress in one member may not necessarily be the best configuration since it may have high stresses in some other members.

For clarity, it must be emphasized that configurations that lead to inducement of the lowest stress in the blocks is desirable since with minimum stress the risk of failure is reduced. This is in contrast with the concept of determining the maximum stress the member could withstand. Configurations that cause maximum stresses in the members should be avoided. It has been experimentally demonstrated that cavity volumes greater than the trough value, the failure of the block is sudden and rather catastrophic making this regime after the trough (plot of strength against ratio of $\left(\mathrm{v}_{\mathrm{c}} / \mathrm{v}_{\mathrm{s}}\right)$ very undesirable $[5,6]$.

\subsection{Effect of Cavity Length}

Generally, the maximum stresses initially fall with the size of cavity (a) between a $=0 \mathrm{~mm}$ and $12.5 \mathrm{~mm}$ and then increases linearly for $\mathrm{a} \geq 12.5 \mathrm{~mm}$. For the ratio $1: 2$ at all the web thickness except $50 \mathrm{~mm}$. for $50 \mathrm{~mm}$, the decrease extended to the point $\mathrm{a}=50 \mathrm{~mm}$ after which there was a continuous increase (Figure 5). The results showed that despite the web thickness the larger the cavity the larger the stress. This is theoretical; however, variation of end conditions may change this largely. There is a noted change in compressive strength as the volume of cavity expressed as a ratio of the volume of solid part is increased. This trend is rather interesting in that as the cavity volume increases the strength decreases to a minimum and then begins to increase. This would appear to be anomalous, as one would generally expect the strength to continue to decrease with 
increasing cavity volume. The observed trend may be ascribed to some sort of size effect $[5,6]$.

At an end-web to centre-web ratio of one (Fig. 6), the curves at various thicknesses are nearly Gaussian with sharp peaks that reduce with increase in web thickness. There is no immediate explanation for the rise in maximum stress between $\mathrm{a}=0 \mathrm{~mm}$ and $\mathrm{a}=$ $100 \mathrm{~mm}$. The lowest of the maximum stresses occur between $\mathrm{a}=150 \mathrm{~mm}$ and $\mathrm{a}=200 \mathrm{~mm}$.

The effects of cavity size on the lowest stresses are shown in Figures 7 and 8. Again, as the size increases the stresses increase for all thicknesses except for $50 \mathrm{~mm}$. At a $=50 \mathrm{~mm}$, there is a sharp fall (Figure 7) creating a global minimum of $0.5 \mathrm{~N} / \mathrm{mm}^{2}$ for $\mathrm{t}=50 \mathrm{~mm}$ and a ratio of $1: 1$. There was no such fall at $t=50 \mathrm{~mm}$ for the ratio 1: 2; rather a decrease occurs along the curve of $\sigma_{\min }$ versus size, a $(\mathrm{mm})$ at $\mathrm{t}=25 \mathrm{~mm}$ and a $=$ $100 \mathrm{~mm}$.

\section{CONCLUSION}

The simulation of the distribution of maximum stresses in sandcrete blocks of different configurations under the assumption of uniformly distribution load from the test thin slab with the blocks behaviourally similar to concrete is obviously approximate. However, it can offer useful insight about the

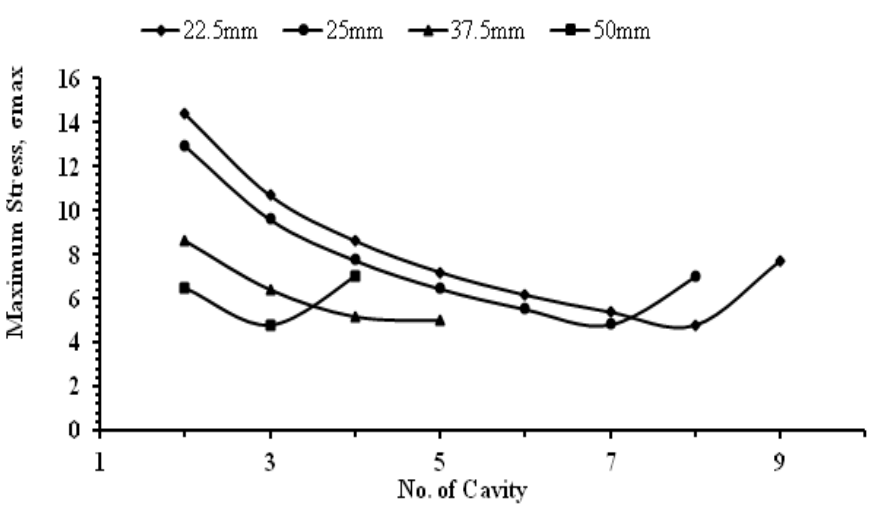

Fig. 1: Maximum stress, $\sigma$ max against the number of cavity where end-web to centre web ratio is $1: 2$

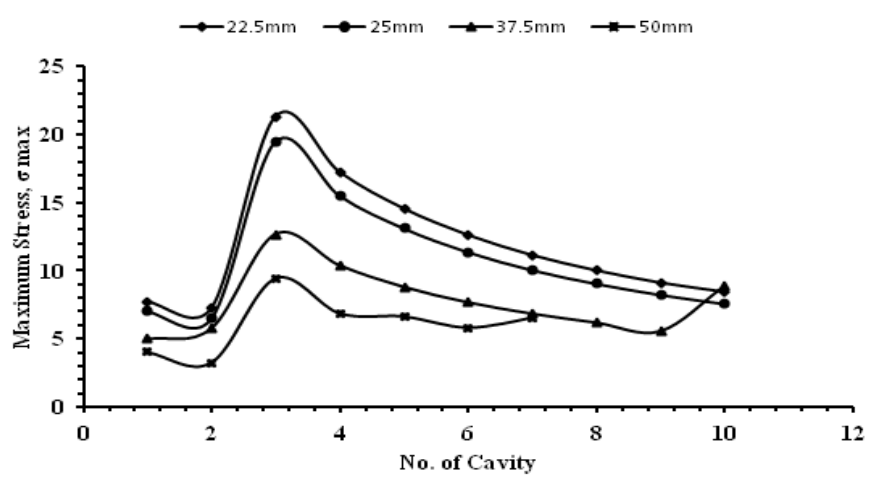

Fig. 2: Maximum stress against the number of cavity for the end-web to centre ratio of 1:1 configurations that could induce maximum stresses in blocks and so should be avoided. It could also yield the configurations that should yield the lowest stresses.

From the analysis, the following conclusions are deduced:

(1) Construction of blocks with end-web to centreweb ratio of $1: 1$ and thickness equal to $22.5 \mathrm{~mm}$ should be avoided as it induces a maximum stress in the sandcrete blocks.

(2) The optimum choice that results in the lowest stress $\left(0.5 \mathrm{~N} / \mathrm{mm}^{2}\right)$ is where the thickness is $50 \mathrm{~mm}$ for an end-web to centre-web ratio of 1:1. However, it creates high stress in other members. Overall, the best configurations for the ratios of $1: 1$ and $1: 2$ are 2 and 3 cavities respectively with $50 \mathrm{~mm}$ shell thickness. The corresponding stresses are $3.3 \mathrm{~N} / \mathrm{mm}^{2}$ and $4.8 \mathrm{~N} / \mathrm{mm}^{2}$ respectively.

(3) The distribution of the maximum and minimum stress for various numbers of cavities and the end to centre web ratios of 1: 1 and 1:2 are Gaussian and polynomial respectively. On the other hand, the distribution of the lowest stresses does not generally fit into any form.

However, it is suggested that these results be verified with data from laboratory experiments.

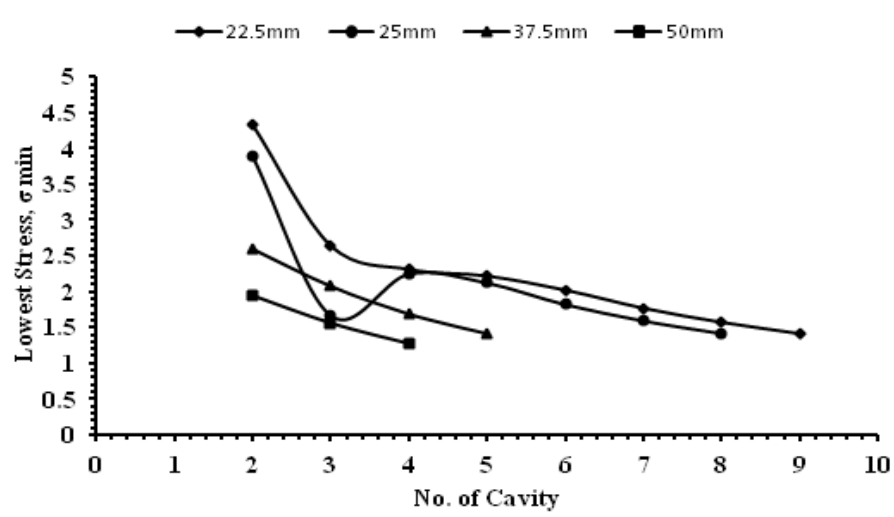

Fig. 3: Lowest stress against the number of cavity for end-web to centre-web of $1: 2$

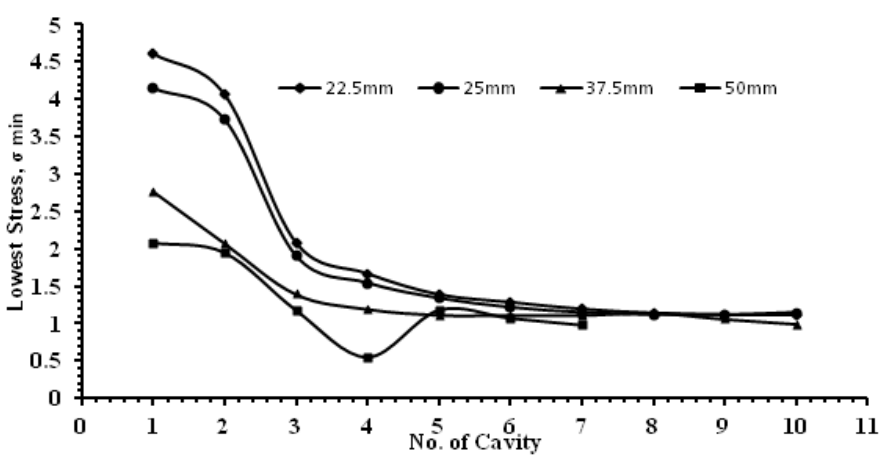

Fig. 4: Lowest Stress against number of cavity for the end-web to centre-web ratio of 1:1

Volume 35, No.2, April 2016 


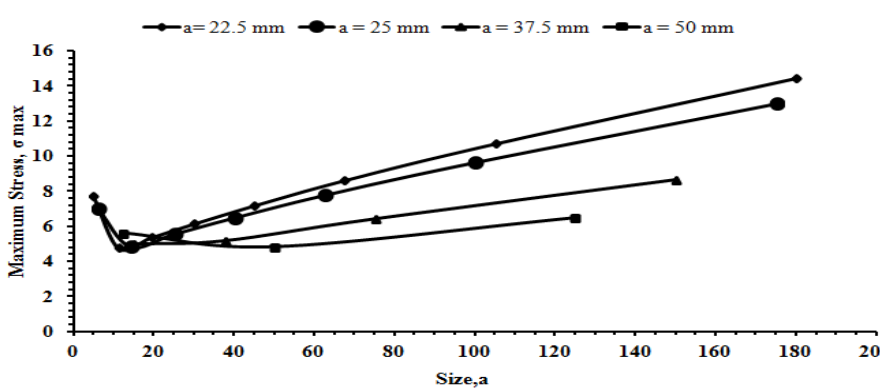

Fig. 5: Maximum stress, ormax against size, a where end-web to centre-web is 1:2

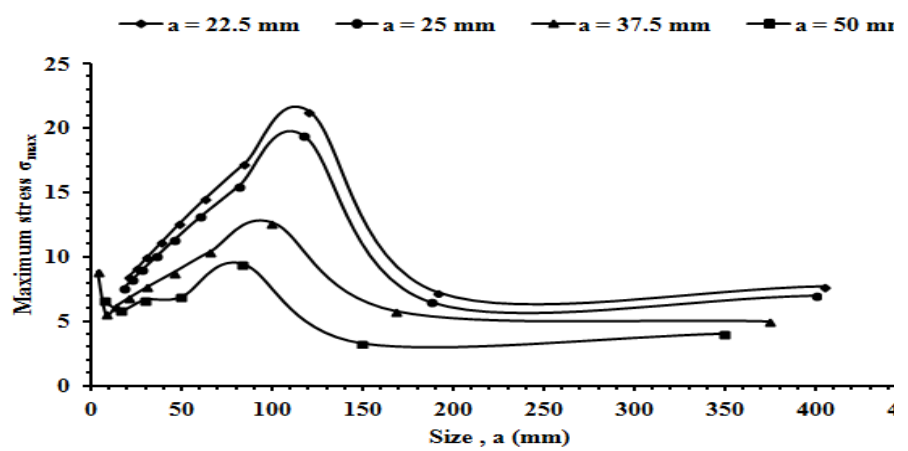

Fig 6: Maximum stressagainst size, a for the end-web to centre web ratio of $1: 1$

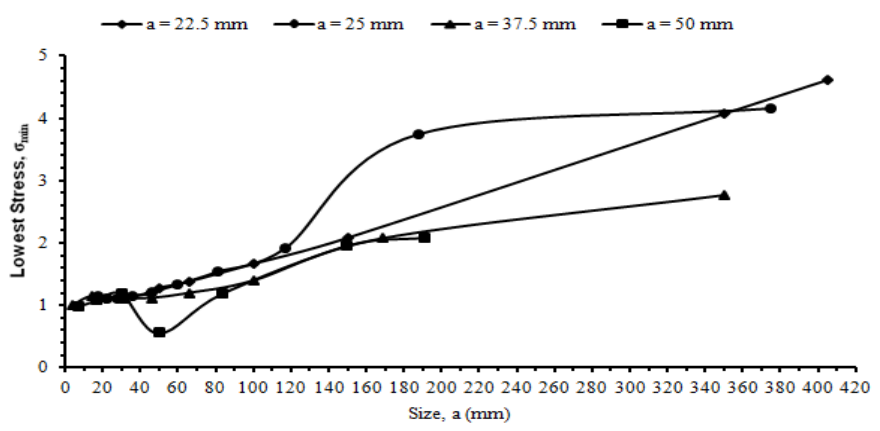

Fig. 7 Lowest stress against size, a for the end-web to centre-web ratio is $1: 1$

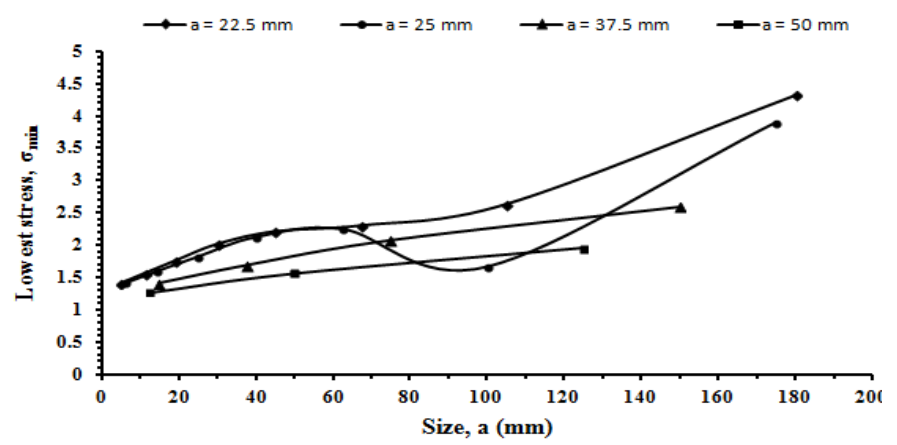

Fig 8: Lowest stress against size, a for the end-web to centre-web of 1:2

\section{REFERENCES}

[1] Barry, R.. The construction of building Crosby Lockwood; London, Vol. 1, 1969, 54 - 55, 94.

[2] Hijab, M; Halilu, A. H. and Hadi, A. A. Compressive strength of marketed sandcrete blocks produced in
Yola, Nigeria. Journal of Engineering and Applied Science 2, 2010, $74-81$.

[3] Anosike, M.N. and Oyebade, A.A.. Sandcrete blocks and quality management in Nigeria Building Industry. J. of Engineering Project and Production Management. 2(1), 2012, 37- 46.

[4] Udoeyo, F. F., Brooks, R., Udo-Inyang, P and Kehinde, A.M.. Influence of specimen geometry on the strength of laterized concrete. IJRRAS, 3(1), 2010, 1 -10.

[5] Ezeokonkwo, J. C.. Uniaxial Compressive Strength of Sandcrete hollow blocks and its dependence on geometry. M. Eng. Thesis. Dept. of Civil Engineering, UNN, 1988, pp. 44- 47.

[6] Ezeokonkwo, J.C.. Optimization of cavity size in hollow sandcrete blocks. J. of Engineering Trends in Engineering and Applied Sci. 3(1), 2012, 185 188.

[7] Reynolds, C., Steedman, E., James, C. and Threlfall, A. J. Reinforced concrete Desgner's Handbook. 2008, Taylor and Francis, P. 63.

\section{APPENDIX: EXAMPLE OF CALCULATIONS FOR 1:2 SERIES ARRANGEMENT THICKNESS $22.5 \mathrm{~mm}-45 \mathrm{~mm}-22.5 \mathrm{~mm}$}

$\mathrm{W}$ is the Typical load carried by the hollow sandcrete block and obtained from Reynolds et al. [7].The supports are treated as simply support and fixed support and depends on the position of the cavity. $\mathrm{R}$ is reaction at support. The value $\mathrm{k}$ is a ratio and how to obtain it is given below.

$$
\begin{aligned}
& 45 \underbrace{R_{11}^{R 2}}_{190.001} \\
& k=\frac{L y}{L x}=\frac{225}{225}=1 \\
& R_{3}=\frac{5}{8} k\left(1-\frac{3}{8} k\right) w l x 2 \\
& =\frac{5}{8} \times 1\left(1-\frac{3}{8}\right) \times 1.8469 \times 2252 \\
& =36523.2 \mathrm{~N} \\
& R_{1}=\frac{3}{5} R_{3}=\frac{3}{5} \times 36523.2=21913.9 \mathrm{~N} \\
& R_{2}=R_{4}=\frac{3}{16} K^{2} w l x^{2} \\
& =\frac{3}{16} \times 1.0^{2} \times 1.8469 \times 2252 \\
& =17531.1 \mathrm{~N}
\end{aligned}
$$




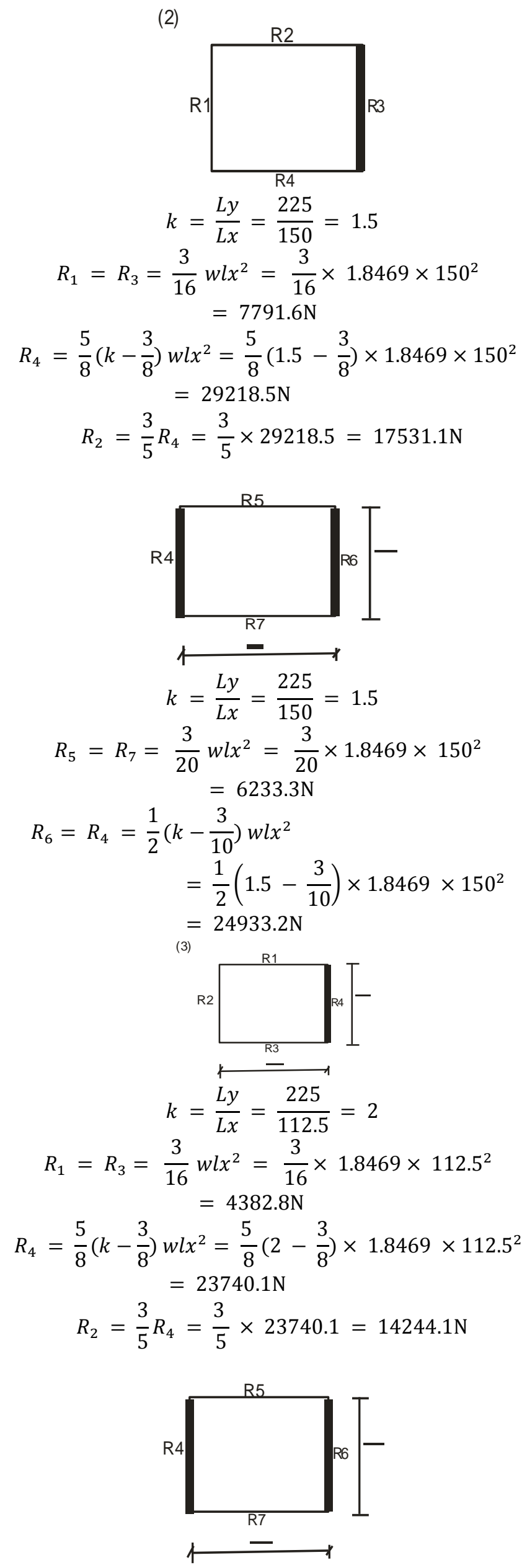

$$
\begin{aligned}
& k=\frac{L y}{L x}=\frac{225}{112.5}=2 \\
& R_{5}=R_{7}=\frac{3}{20} w l x^{2}=\frac{3}{20} \times 1.8469 \times 112.5^{2} \\
& =3506.2 \mathrm{~N} \\
& R_{4}=R_{6}=\frac{1}{2}\left(k-\frac{3}{10}\right) w l x^{2} \\
& =\frac{1}{2}\left(2-\frac{3}{10}\right) \times 1.8469 \times 112.5^{2} \\
& =19868 \mathrm{~N} \\
& \text { (4) }
\end{aligned}
$$

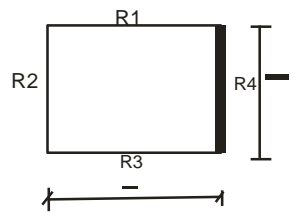

$$
\begin{aligned}
& k=\frac{L y}{L x}=\frac{225}{90}=2.5 \\
& R_{1}=R_{3}=\frac{3}{16} w l x^{2}=\frac{3}{16} \times 1.8469 \times 90^{2}=2805 \mathrm{~N} \\
& R_{4}=\frac{5}{8}\left(k-\frac{3}{8}\right) w l x^{2}=\frac{5}{8}\left(2.5-\frac{3}{8}\right) \times 1.8469 \times 90^{2} \\
& =19868.6 \mathrm{~N} \\
& R_{2}=\frac{3}{5} R_{4}=\frac{3}{5} \times 19868.6=11921.2 \mathrm{~N} \\
& k=\frac{L y}{L x}=\frac{225}{90}=2.5 \\
& R_{5}=R_{7}=\frac{3}{20} w l x^{2}=\frac{3}{20} \times 1.8469 \times 90^{2} \\
& =2244.0 \mathrm{~N} \\
& R_{4}=R_{6}=\frac{1}{2}\left(k-\frac{3}{10}\right) w l x^{2} \\
& =\frac{1}{2}\left(2.5-\frac{3}{10}\right) \times 1.8469 \times 90^{2} \\
& =16455.9 \mathrm{~N} \\
& k=\frac{L y}{L x}=\frac{225}{90}=2.5 \\
& R_{8}=R_{10}=2244 \mathrm{~N} \\
& R_{6}=R_{9}=16455.9 \mathrm{~N} \\
& k=\frac{L y}{L x}=\frac{225}{75}=3
\end{aligned}
$$
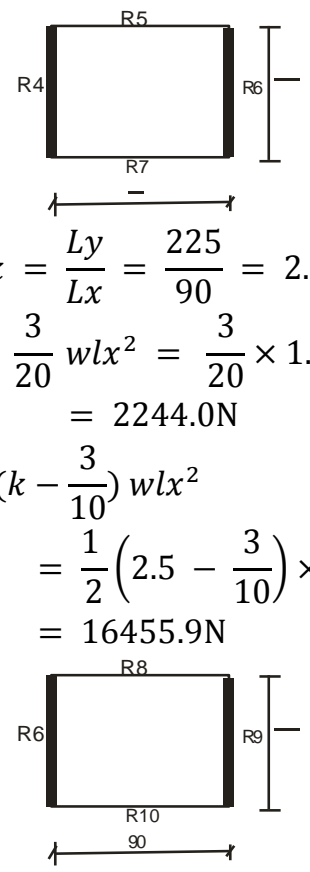
Theoretical Investigation Of STResSes Distributions in Hollow SANDCRETe BlockS .... J. C. Agunwamba, et al

\begin{tabular}{|c|c|c|c|c|c|c|}
\hline \multirow[b]{2}{*}{$S / N$} & \multirow[b]{2}{*}{ Cavity Volume } & \multirow[b]{2}{*}{$t$} & \multicolumn{2}{|c|}{ Dimension } & \multirow{2}{*}{$\begin{array}{c}\text { No. of } \\
\text { Suppo } \\
\text { rt }\end{array}$} & \multirow[b]{2}{*}{ Stress, $\sigma$} \\
\hline & & & $\mathrm{a}$ & $\mathrm{b}$ & & \\
\hline 1 & 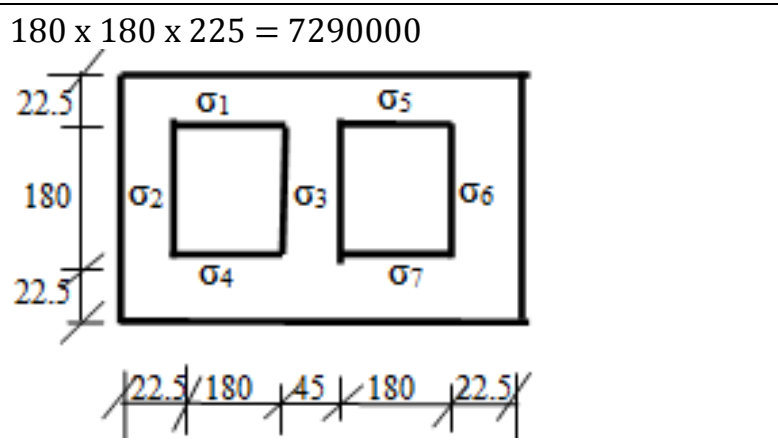 & & 180 & 180 & & $\begin{array}{l}\sigma_{1}=\frac{21913.9}{225 \times 22.5}=4.32867 \\
\sigma_{2}=\sigma_{4}=\frac{36531.1}{180 \times 22.5}= \\
14.42892 \\
\sigma_{3}=2\left(\frac{36523.2}{225 \times 22.5}\right)=14.42892 \\
\sigma_{5}=\sigma_{7}=\sigma_{2}=\sigma_{4}=4.32867 \\
\sigma_{6}=\sigma_{1}=4.32867\end{array}$ \\
\hline 2 & 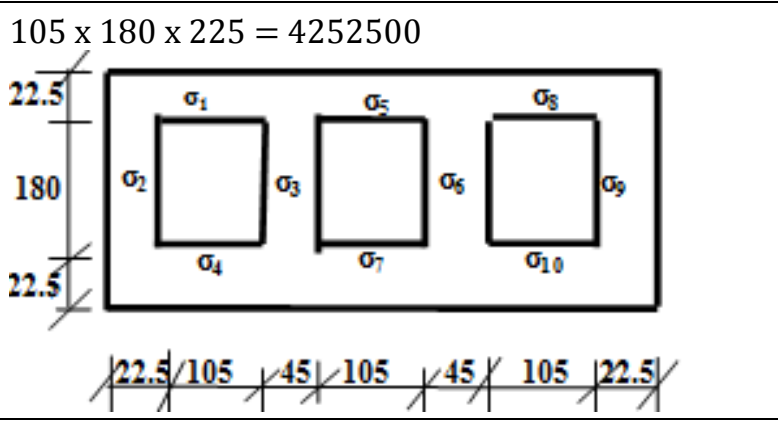 & & 105 & 180 & 4 & $\begin{array}{l}\sigma_{1}=\sigma_{3}=\frac{7791.6}{105 \times 22.5}=3.29803 \\
\sigma_{2}=\frac{17531.1}{225 \times 2.5}=3.46293 \\
\sigma_{4}=\frac{29218.5}{225 \times 22.5}+\frac{24933.2}{225 \times 22.5} \\
=10.69663 \\
\sigma_{5}=\sigma_{7}=\frac{24933.2}{105 \times 22.5}=2.63843 \\
\sigma_{6}=\frac{24933.2}{225 \times 22.5}=4.92508 \\
\sigma_{8}=\sigma_{10}=\sigma_{1}=\sigma_{3}=3.29803 \\
\sigma_{9}=\sigma_{2}=3.46293\end{array}$ \\
\hline 3 & 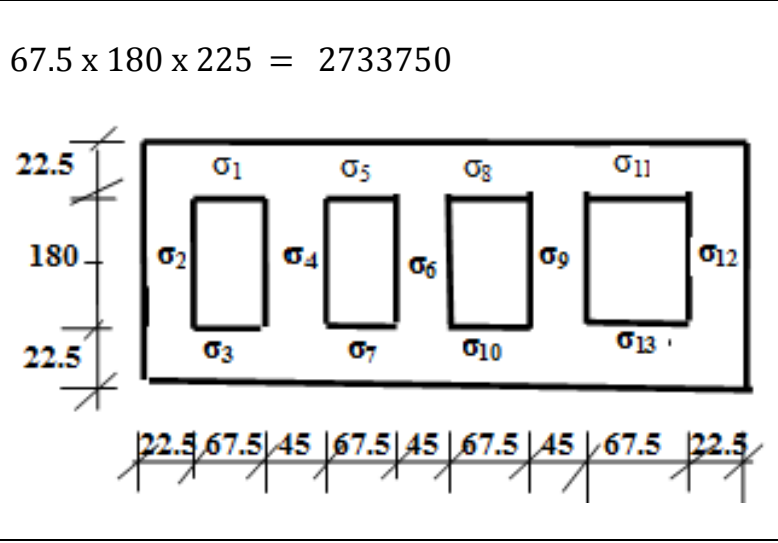 & & 67.5 & 180 & 5 & $\begin{array}{l}\sigma_{1}=\sigma_{3}=\frac{4382.8}{67.5 \times 22.5}=2.88579 \\
\sigma_{2}=\frac{14244.1}{225 \times 22.5}=2.81365 \\
\sigma_{4}=\frac{23740.1}{225 \times 22.5}+\frac{19868.6}{225 \times 22.5} \\
=8.61406 \\
\sigma_{5}=\sigma_{7}=\frac{3506.2}{6.5 \times 2.5}=2.30861 \\
\sigma_{6}=2\left(\frac{19868.6}{225 \times 22.5}\right)=7.84932 \\
\sigma_{8}=\sigma_{10}=\sigma_{5}=\sigma_{7}=2.30861 \\
\sigma_{9}=\sigma_{4}=8.61406 \\
\sigma_{11}=\sigma_{13}=\sigma_{1}=\sigma_{3}=2 . \\
88579 \\
\sigma_{12}=\sigma_{2}=2.81365\end{array}$ \\
\hline 4 & 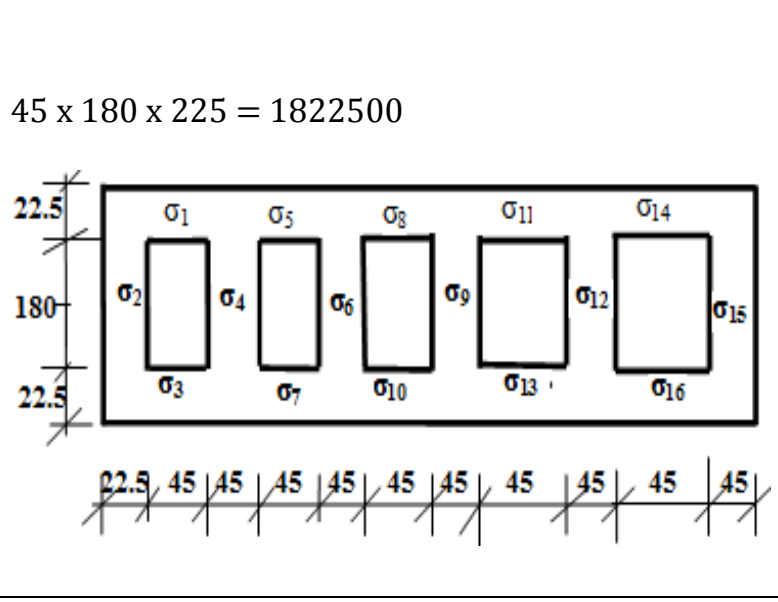 & & 45 & 180 & 6 & $\begin{array}{l}\sigma_{1}=\sigma_{3}=\frac{2805}{45 \times 22.5}=2.7704 \\
\sigma_{2}=\frac{11921.2}{225 \times 22.5}=2.35480 \\
\sigma_{4}=\frac{19868.6}{225 \times 22.5}+\frac{16455.9}{225 \times 22.5} \\
=7.17521 \\
\sigma_{5}=\sigma_{7}=\frac{2244}{45 \times 22.5}=2.21630 \\
\sigma_{6}=2\left(\frac{16455.9}{225 \times 2.5}\right)=6.50110 \\
\sigma_{8}=\sigma_{10}=\frac{2244}{45 \times 22.5}=2.21630 \\
\sigma_{9}=\sigma_{6}=6.50110 \\
\sigma_{11}=\sigma_{13}=\sigma_{5}=\sigma_{7}=2.21630 \\
\sigma_{12}=\sigma_{4}=7.17521 \\
\sigma_{14}=\sigma_{16}=\sigma_{1}=\sigma_{3}=2.7704 \\
\sigma_{15}=\sigma_{2}=2.35480\end{array}$ \\
\hline
\end{tabular}

